\title{
Transient emission of selected CRTS Cataclysmic Variables
}

\author{
H. Szegedi ${ }^{* 1}$, A. Odendaal ${ }^{1}$, P.J. Meintjes ${ }^{1}$, B. van Soelen ${ }^{1}$, J.P. Marais ${ }^{1}$, A.F. \\ Rajoelimanana $^{1}$, M.M. Nyamai ${ }^{1}$, R.J. Britto ${ }^{1}$, L. Hanlon ${ }^{2}$, D. Murphy ${ }^{2}$, A. \\ Martin-Carrillo ${ }^{2}$, M. Motsoaledi ${ }^{3}$ and J.R. Thorstensen ${ }^{4}$ \\ ${ }^{1}$ University of the Free State, Bloemfontein, South Africa \\ ${ }^{2}$ University College Dublin, Dublin, Ireland \\ ${ }^{3}$ University of Cape Town, Cape Town, South Africa \\ ${ }^{4}$ Dartmouth College, Hanover, United States of America \\ E-mail: szegediH@ufs.ac.za
}

\begin{abstract}
We present optical photometric and spectroscopic observations of three SU UMa-type dwarf novae, i.e. AR Pic, QW Ser and V521 Peg, conducted in 2016 and 2017. These sources were selected from the Catalina Real-Time Transient Survey and observed during quiescence, outburst (AR Pic and QW Ser) and superoutburst (V521 Peg). For AR Pic, strong flickering in the light curves and an asymmetric double-peaked $\mathrm{H} \beta$ emission line in the spectra, confirmed the presence of a very active hot spot. During outburst, detected on 18 February 2017, it exhibited a $\sim 3.3$ magnitude brightening. The projected velocity of the inner edge of the accretion disc is $\sim 2000 \mathrm{~km} \mathrm{~s}^{-1}$. An outburst of QW Ser was detected on 8 August 2016. Absorption lines, characteristic of an optically thick disc during outburst, were detected and an outflow velocity of $\sim 1000 \mathrm{~km} \mathrm{~s}^{-1}$ was determined. Optical spectra during the superoutburst of V521 Peg were obtained for the first time, confirming the superoutburst nature of the outburst, as also observed in other SU UMa-type dwarf novae. The superoutburst was detected on 1 September 2017. Welldefined superhumps were observed, with an average superhump period of $P_{\mathrm{sh}} \sim 1.48 \mathrm{hr}$. A mass ratio of $q \sim 0.14$ was determined from the period excess of $\varepsilon \sim 3 \%$.
\end{abstract}

The Golden Age of Cataclysmic Variables and Related Objects IV

11-16 September, 2017

Palermo, Italy

* Speaker. 


\section{Introduction}

Cataclysmic variables (CVs) are compact binary systems that consist of a white dwarf (WD) primary accreting material usually from a red dwarf ( $\mathrm{K}$ or $\mathrm{M}$ spectral type) secondary (but also from a WD donor in CVs with ultra-short orbital periods), due to Roche-lobe overflow ([1], [2] and [3]). The orbital periods of CVs and related close binary systems are from $5.36 \mathrm{~min}$ to 5.714 days [4]. In non-magnetic CVs (NMCVs, with magnetic field $B<1 \mathrm{MG}$ ), matter streams through the inner Langragian point L1 and accretes onto the WD surface via an accretion disc. A hot spot is formed where the stream impacts the accretion disc. In the accretion disc, viscosity acts on the material, causing an outward transport of angular momentum, enabling the material to move inwards [5]. Dwarf novae (DNe), a subclass of NMCVs, experience outbursts due to a release of gravitational energy caused by a temporary increase in the mass transfer rate in the accretion disc (see [2] and [6] for a review).

DNe are classified as Z Cam, SU UMa or U Gem systems, based on the morphology of their light curves (e.g. [2], p. 28). All DNe experience normal outbursts, which last for $\sim 2$ to 3 days ( $\sim 2-8$ mag increase [7]), but only SU UMa DNe experience superoutbursts that last $\sim 5$ times longer than normal outbursts ( $\sim 10-14$ days) [8]. Superhumps, with amplitudes of $\sim 0.2$ to 0.3 mag [9], are visible in the light curves during superoutbursts and have periods a few percent longer than the orbital period [10]. SU UMa stars alternate between more frequent normal outbursts and less frequent superoutbursts. Both types of outbursts are initially caused by a thermal-viscous instability in the accretion disc, but Whitehurst [8] discovered that it is a tidal instability in the accretion disc of binary systems with a mass ratio $q<0.25$ that drives superoutbursts. Here $q$ represents the ratio of companion mass to WD mass. Hirose and Osaki [11] further showed that accretion discs in binary systems with $q<0.25$ become tidally unstable when the outer disc exceeds a critical radius $R_{\mathrm{cr}} \simeq 0.46 a, a$ being the binary separation, and that the instability is caused by a parametric resonance, with a ratio of 3:1, between the particle orbit and the binary orbit.

During quiescence, the disc is in a cool, low-viscosity state. As material accumulates, the disc's temperature gradually increases, causing an increase in viscosity. In time, the disc will become fully ionized [6], allowing the material to interact with the magnetic field lines. Turbulence driven by magnetohydrodynamic instabilities provides a sustainable mechanism for the transport of angular momentum [12]. When angular momentum is transported from the inner parts of the disc to the outer parts, the material starts to spiral toward the WD at a faster rate. The "frozen-in" magnetic field lines are dragged along the Keplerian flow of material ([13] and [14]) and become unstable to reconnection. Magnetic turbulence can lead to the formation of magnetic eddies. Eventually the disc will be in a hot, highly viscous state and enhanced accretion due to a magneto-gravitational instability will give rise to a normal outburst ([15] and [16]).

Non-periodic flickering in a light curve is attributed to the swirling motion of the magnetic eddies, displaying a characteristic red-noise behaviour defined by the Kolmogorov $E(k) \propto k^{-5 / 3}$ law (e.g. [15] and references therein), as well as temperature changes in the hot spot due to density fluctuations in the stream [17].

The outward transport of angular momentum in the disc causes the radius of the outer disc to gradually increase after every normal outburst. When the disc reaches the critical radius it becomes tidally unstable against non-axisymmetric perturbation and a slowly precessing, eccentric 
disc develops. In the co-rotating reference frame of the system, the disc precesses in the direction opposite to the orbital motion, but from the observer's reference frame the eccentric disc rotates in the same direction as the orbital motion [11], with a precession period significantly longer than the orbital period. During every orbital cycle, the tidal influence of the secondary will deform the disc and as angular momentum from the outer parts of the disc is removed by the tidal torque of the secondary, the disc's edge contracts. The surface density at the outer edge is hence pushed above a critical density, where no low-viscosity cool state exists, and a superoutburst is triggered as the disc is kept in a hot state (see [18] for a full analytical treatment and summary). This also explains why a superoutburst is in a prolonged maximum state and is slower to decline to quiescence compared to a normal outburst. The prolonged maximum is observable as a sloping plateau in the light curve. The observed superhump represents the varying amount of tidal energy released by the deformation through an orbital cycle and the moments of maximum tidal stress in the disc are separated by a time frame (superhump period) a few percent longer than the orbital period [8]. The superoutburst will come to an end when the contracting disc's non-axisymmetric nature decays, reducing the tidal torque, and the disc finally transitions back to a low-viscosity state as a cooling wave propagates inwards. When the system settles back into quiescence, a new superoutburst cycle ("supercycle") commences.

The superhump period $P_{\mathrm{sh}}$ is the beat period between the orbital period $P_{\mathrm{orb}}$ and the precession period $P_{\text {prec}}$, and expressed as

$$
\frac{1}{P_{\mathrm{sh}}}=\frac{1}{P_{\text {orb }}}-\frac{1}{P_{\text {prec }}}
$$

Osaki [19] determined a ratio between the precession frequency $\omega_{\text {prec }}$ and the orbital frequency $\omega_{\text {orb }}$ which is given by

$$
\frac{\omega_{\mathrm{prec}}}{\omega_{\mathrm{orb}}}=\frac{3}{4} \frac{q}{\sqrt{1+q}}\left(\frac{R_{d}}{a}\right)^{3 / 2},
$$

which can be simplified and expressed in terms of the orbital period $P_{\text {orb }}$ and precession period $P_{\text {prec }}$ as

$$
\frac{P_{\text {orb }}}{P_{\text {prec }}} \approx \frac{0.233 q}{\sqrt{1+q}}
$$

where it is assumed that the outer disc radius is $R \approx R_{\mathrm{cr}} \simeq 0.46 a$. The period excess $\varepsilon$ is defined as

$$
\varepsilon=\frac{\Delta P}{P_{\text {orb }}}=\frac{P_{\text {sh }}-P_{\text {orb }}}{P_{\text {orb }}} .
$$

The relation between the period excess and the mass ratio can be derived from the precession period in Equation (1.3):

$$
\varepsilon \approx \frac{0.23 q}{1+0.27 q} .
$$

The focus of this discussion is on three SU UMa DNe, namely AR Pic, QW Ser and V521 Peg, that were selected from the Catalina Real-Time Transient Survey (CRTS) for follow-up studies. Each source exhibited very different transient behaviour during our optical observations, which are all characteristic of SU UMa sources. The method by which these sources were selected from the CRTS and what was previously known about them, will be discussed in the following section. In section 3, it is explained how the optical photometric and spectroscopic observations were conducted, followed by the associated results in section 4 and the concluding remarks in section 5 . 


\section{CRTS and the selection criteria}

The Catalina Real-Time Transient Survey ${ }^{1}$ (CRTS) is the combined effort of three separate sub-surveys, namely the Catalina Schmidt Survey (CSS), the Mount Lemmon Survey (MLS) and the Siding Spring Survey (SSS) [20]. The aim of the survey is to identify near-Earth asteroids, as well as sources manifesting transient behaviour, e.g. CVs, supernovae and blazars [21]. At the start of operation in 2007, the CRTS initially consisted of three telescopes run by the sub-surveys, which, combined, covered $\sim 30000 \mathrm{deg}^{2}$ of sky in the declination range $-75^{\circ}<\delta<65^{\circ}$. However, the SSS, the southern hemisphere counterpart of the CRTS, ended in July 2013. To date, the CRTS has identified the largest sample of CVs from a single survey (1440 CVs by March 2018).

A sample of CV candidates were selected from the CRTS database for follow-up studies. The selection criteria included sources that exhibit variability of more than 2 magnitudes, enabling the study of transient emission. The candidates also had to have $V$-magnitudes less than 19 and declination values less than $+30^{\circ}$. This ensured that the sample was observable from South Africa and within the observable limits of the photometric and spectroscopic telescope systems used.

One of the aims of the study is to determine the energy output of CVs during outbursts. The light curves, provided by the CRTS database, were therefore used to select sources that experienced outbursts frequently. This required that the sources should have been observed for more than a year by CRTS. Additional light curves from the ASAS-3 (All Sky Automated Survey) [22] were used for supplementary outburst detections. ASAS-3, located at the Las Campanas Observatory in Chile, surveyed the southern skies $\left(\delta<+28^{\circ}\right)$ from 2000 until 2009. It detected sources with $V$ brighter than 15 mag. It is beneficial to use these light curves, since ASAS-3 detected outbursts pre-CRTS and concurrently with the CRTS data, therefore detecting outbursts possibly missed by CRTS.

The three SU UMa sources discussed in this paper, AR Pic, QW Ser and V521 Peg, were part of the selected sample. Their $V$-magnitudes during outburst $\left(V_{\mathrm{o}}\right)$ and quiescence $\left(V_{\mathrm{q}}\right)$ (obtained from CRTS), as well as their known orbital periods and average superhump periods are given in Table 1. A brief description of each source is given below.

Table 1: Properties of the three selected SU UMa sources. $V_{\mathrm{o}}$ and $V_{\mathrm{q}}$ are the $V$-magnitudes during outburst and quiescence, respectively, obtained from CRTS. $P_{\mathrm{orb}}$ is the orbital period and $P_{\mathrm{sh}}$ is the superhump period, as obtained from the outburst catalogue of cataclysmic variables [7], accessible through the CDS Vizier website $^{*}[23]$.

\begin{tabular}{lllllll}
\hline Name & RA (J2000) & Dec. (J2000) & $V_{\mathrm{o}}$ & $V_{\mathrm{q}}$ & $P_{\text {orb }}(\mathrm{hr})$ & $P_{\text {sh }}(\mathrm{hr})$ \\
\hline AR Pic & $05: 49: 45.41$ & $-49: 21: 56.4$ & 13.2 & 16.9 & 1.93 & 1.99 \\
QW Ser & $15: 26: 13.99$ & $+08: 18: 02.2$ & 12.6 & 17.9 & 1.79 & 1.85 \\
V521 Peg & $22: 21: 44.80$ & $+18: 40: 08.4$ & 12.3 & 17.3 & 1.44 & 1.47 \\
\hline *http://cds.u-strasbg.fr/ & & & & &
\end{tabular}

\subsection{AR Pic}

AR Pictoris (AR Pic, CTCV J0549-4921) was identified as a CV candidate in the Calán-Tololo Survey [24] and classified as a DN by Tappert et al. [25]. Imada et al. [26] confirmed the SU

\footnotetext{
${ }^{1}$ http://crts.caltech.edu/
} 
UMa nature of AR Pic when superhumps were observed during a superoutburst in April 2006. In quiescence, the source is faint with $V \sim 16.9$ mag and it has an average outburst amplitude of $\sim 4$ mag [21]. In the CRTS and ASAS-3 light curves, a number of outbursts were detected. The source generally has short outbursts that last only a day. It is therefore assumed that the heating wave does not propagate to the disc's edge and is shut down fairly quickly by a cooling wave, initiated by a cold region sucking material from the hotter region. The duration of the observed superoutbursts vary between 6 and 10 days (see e.g. [27], Table 304) and the supercycle length is approximately $750-800 \mathrm{~d}$. The orbital ephemeris was determined as

$$
T_{0}(H J D)=2451118.8019(22)+0.080218(70) E
$$

where $T_{0}(H J D)$ is the heliocentric Julian date of minimum light in the light curve [25]. In the folded light curve obtained by Tappert et al. (see e.g. [25] Figure 4) two humps of unequal amplitude were visible during one orbital period, when the source was in quiescence. This is attributed to the hot spot being at inferior and superior conjunction in the system, typically seen in low mass transfer rate DNe. The light curves also showed no eclipse, indicating that the system has a low to intermediate inclination. Light curves for an outburst in 1996 showed no periodic features, but moderate flickering.

\subsection{QW Ser}

QW Serpentis (QW Ser, TmzV46) was discovered by Takamizawa [28], who suggested that it may be a DN, and Schmeer [29] confirmed this classification by observing a normal outburst in 1999. Nogami et al. [30] observed four superoutbursts, which occurred between 2000 and 2003, and confirmed that QW Ser was a SU UMa-type DN. During quiescence the source is fairly faint with $V \sim 18$ mag. Nogami et al. [30] determined an absolute maximum magnitude of $M_{\mathrm{V}}=5.2 \pm$ 0.2 , giving an estimated distance of $380( \pm 60)$ pc. Previous outbursts, reported by Takamizawa and detected by ASAS-3, show that an outburst lasted $\sim 2-3 \mathrm{~d}$ and a superoutburst lasted $\sim 5-15 \mathrm{~d}$ (see e.g. [30] Table 4). The outburst cycle length is $\sim 50 \mathrm{~d}$ and the supercycle length is $\sim 220-270$ $\mathrm{d}$ since 1999. An ephemeris of the orbital period has not been established.

Mass ratios have been determined by Olech et al. [31], $q \sim 0.15$, Patterson et al. [32], $q \sim$ 0.147 , and Pearson [33], $q \sim 0.144$. No eclipses were observed during quiescence, but a doublepeaked $\mathrm{H} \alpha$ line, with a peak separation of $1100 \mathrm{~km} \mathrm{~s}^{-1}$, was resolved in spectroscopic observations [32]. This indicated that the system must have an intermediate inclination.

\subsection{V521 Peg}

V521 Pegasi (V521 Peg, HS 2219+1824) was first classified as a CV candidate based on spectroscopic data from the Hamburg Quasar Survey [34]. Rodríguez-Gil et al. [35] was able to identify the source as a SU UMa-type DN through follow-up optical photometric and spectroscopic observations, conducted from 2000 until 2003 during quiescence and superoutburst. The authors determined a mass ratio of $0.14 \leq q \leq 0.19$ and a superoutburst amplitude of $\sim 5.5 \mathrm{mag}$. The optical spectrum displayed broad Balmer absorption troughs, in which the $\mathrm{H} \beta$ and $\mathrm{H} \gamma$ emission lines were embedded. These troughs were attributed to the photospheric spectrum of a WD with an effective temperature of $13000 \mathrm{~K} \leq T_{\text {eff }} \leq 17000 \mathrm{~K}$, at a distance $180 \mathrm{pc} \leq d \leq 230 \mathrm{pc}$. 
A peculiar narrow emission line within the double-peaked $\mathrm{H} \alpha$ line was also observed, which is ascribed to chromospheric emission from the irradiated inner hemisphere of the donor. Kato et al. (e.g [36] and [37]) observed superoutbursts in 2012 and 2013. To date no normal outbursts have been observed for this source.

\section{Observations}

Follow-up optical photometric and spectroscopic observations were conducted at the Boyden Observatory and the Sutherland station of the South African Astronomical Observatory (SAAO). Approximately $60 \%$ of the spectroscopic observations were carried out simultaneously with the photometric observations. The details of all the observations performed for each source are summarized in Tables 2 and 3.

\subsection{Boyden Observatory}

At the Boyden Observatory, located $26 \mathrm{~km}$ north-east of Bloemfontein, two telescopes were utilized for photometric observations. QW Ser and V521 Peg were observed in quiescence and during outburst with the UFS-Boyden 1.52-m Cassegrain telescope. The CCD provides a fieldof-view (FOV) of 2.5 arcmin $\times 3.7$ arcmin. The filter wheel has Johnson UBVRI and C (Clear, white light) filters available, but only the C-filter was used during observations, as the sources, in quiescence, were just within the telescope's observable magnitude limit.

When QW Ser and V521 Peg experienced outbursts, they were continuously monitored with the on-site Watcher telescope, until they returned to quiescence. Watcher is a 0.4-m Cassegrain robotic telescope, operated by the Space Science Group at the University College Dublin [38]. Observations were conducted with the fitted focal reducer, providing a FOV of $10 \operatorname{arcmin} \times 10$ arcmin [39]. At the time of the QW Ser outburst observations only one filter wheel, accommodating eight filters namely V, R, OIII, $\mathrm{H} \alpha, \mathrm{g}^{\prime}, \mathrm{i}^{\prime}, \mathrm{r}^{\prime}$ and Clear, where part of the telescope system. A second filter wheel, housing Bessel BVRI filters, was later added and used in combination with the first filter wheel during the observations of V521 Peg.

\subsection{South African Astronomical Observatory}

The 1.0-m Cassegrain telescope and SHOC (Sutherland High-speed Optical Cameras), at SAAO, were used for the photometric observations of QW Ser and AR Pic. The CCD provides a FOV of $2.85 \operatorname{arcmin} \times 2.85 \operatorname{arcmin}$ [40]. The system is ideal for high-speed photometry as it can achieve frame rates up to 20 frames per second during normal operations, or even higher with customized settings. Our observations were performed in conventional mode. The available filters included Bessel UBVRI, Sloan $\mathbf{u}^{\prime} \mathrm{g}^{\prime} \mathrm{r}^{\prime} \mathrm{i}^{\prime} \mathrm{z}^{\prime}$ and Stromgren uvby filters. We only observed the source in "white light".

The optical spectroscopic observations for all three sources were conducted with the SAAO 1.9-m telescope and SpUpNIC (Spectrograph Upgrade - Newly Improved Cassegrain). SpUpNIC is mounted at the Cassegrain focus [41]. No filters were used during observations. A CuAr arc lamp was used to obtain wavelength calibration arcs. Gratings 4 and 7, with resolutions of 1.8 $\AA$ and $6.6 \AA$ respectively, were utilized. 
Table 2: The photometric observations. The total observation time is given by $\Delta t$, the exposure time per frame is $t_{\exp }$ and $n_{\text {data }}$ is the number of data frames taken during the observation.

\begin{tabular}{|c|c|c|c|c|c|c|c|c|}
\hline Name & Date & $\begin{array}{l}\text { Telescope/ } \\
\text { Observatory }\end{array}$ & $\begin{array}{l}\text { HJD (Start) } \\
(2450000+)\end{array}$ & Filter & $\begin{array}{c}\Delta t \\
(\mathrm{hr})\end{array}$ & $\begin{array}{l}t_{\exp } \\
(\mathrm{s})\end{array}$ & $n_{\text {data }}$ & Observers* \\
\hline \multirow[t]{4}{*}{ AR Pic } & 2017-02-19 & $1.0-\mathrm{m} / \mathrm{SAAO}$ & 7804.25896 & $\mathrm{C}$ & 2.00 & 5 & 1441 & MM, JRT \\
\hline & $2017-02-22$ & $1.0-\mathrm{m} / \mathrm{SAAO}$ & 7807.40009 & $\mathrm{C}$ & 3.49 & 5 & 2492 & HS \\
\hline & $2017-02-23$ & $1.0-\mathrm{m} / \mathrm{SAAO}$ & 7808.38524 & $\mathrm{C}$ & 2.04 & 5 & 1471 & HS \\
\hline & $2017-02-26$ & 1.0-m/SAAO & 7811.26001 & $\mathrm{C}$ & 4.68 & 5 & 3360 & HS \\
\hline \multirow[t]{12}{*}{ QW Ser } & 2016-08-04 & 1.5-m/Boyden & 7605.20848 & $\mathrm{C}$ & 1.89 & 25 & 238 & MMN, AFR \\
\hline & 2016-08-08 & 1.5-m/Boyden & 7609.20184 & $\mathrm{C}$ & 2.27 & 25 & 303 & JPM, MMN \\
\hline & 2016-08-09 & 1.5-m/Boyden & 7610.19709 & $\mathrm{C}$ & 4.02 & 25 & 550 & RJB, JPM \\
\hline & 2016-08-10 & 1.5-m/Boyden & 7611.27771 & $\mathrm{C}$ & 1.83 & 25 & 257 & JPM, MMN \\
\hline & 2016-08-10 & 0.4-m/Boyden & 7611.18728 & $\mathrm{~g}^{\prime}, \mathrm{r}^{\prime}, \mathrm{i}^{\prime}$ & 3.94 & 10 & 416 & UCD \\
\hline & 2016-08-11 & 1.5-m/Boyden & 7612.22719 & $\mathrm{C}$ & 3.01 & 25 & 391 & JPM, MMN \\
\hline & 2016-08-11 & 0.4-m/Boyden & 7612.31788 & $\mathrm{~g}^{\prime}, \mathrm{r}^{\prime}, \mathrm{i}^{\prime}$ & 0.65 & 30 & 34 & UCD \\
\hline & 2016-08-12 & 1.5-m/Boyden & 7613.21145 & $\mathrm{C}$ & 3.11 & 40 & 255 & JPM \\
\hline & 2016-08-12 & 0.4-m/Boyden & 7613.18743 & $\mathrm{~g}^{\prime}, \mathrm{r}^{\prime}, \mathrm{i}^{\prime}$ & 3.78 & 30 & 151 & UCD \\
\hline & 2016-08-14 & 1.5-m/Boyden & 7615.20306 & $\mathrm{C}$ & 3.53 & 40 & 310 & RJB, JPM \\
\hline & 2016-08-14 & 0.4-m/Boyden & 7615.18757 & $\mathrm{~g}^{\prime}, \mathrm{r}^{\prime}, \mathrm{i}^{\prime}$ & 3.67 & 30 & 152 & $\mathrm{UCD}$ \\
\hline & 2017-03-04 & 1.0-m/SAAO & 7817.53568 & $\mathrm{C}$ & 2.54 & 30 & 306 & JPM \\
\hline \multirow[t]{25}{*}{ V521 Peg } & 2016-08-08 & 1.5-m/Boyden & 7608.52877 & $\mathrm{C}$ & 0.52 & 30 & 62 & JPM, MMN \\
\hline & 2016-08-09 & 1.5-m/Boyden & 7610.47125 & $\mathrm{C}$ & 1.35 & 30 & 159 & RJB, JPM \\
\hline & 2017-09-02 & 1.5-m/Boyden & 7999.29529 & $\mathrm{C}$ & 4.50 & 10 & 1253 & HS \\
\hline & 2017-09-03 & 1.5-m/Boyden & 8000.28121 & $\mathrm{C}$ & 4.50 & 10,20 & 1082 & HS \\
\hline & 2017-09-04 & 1.5-m/Boyden & 8001.27181 & $\mathrm{C}$ & 4.50 & 20 & 791 & HS \\
\hline & 2017-09-04 & 0.4-m/Boyden & 8001.36777 & $\mathrm{~B}, \mathrm{~V}, \mathrm{R}, \mathrm{I}$ & 1.74 & 30 & 110 & UCD \\
\hline & 2017-09-05 & 1.5-m/Boyden & 8002.27583 & $\mathrm{C}$ & 4.50 & 20 & 732 & HS \\
\hline & 2017-09-05 & 0.4-m/Boyden & 8002.28505 & $\mathrm{~V}, \mathrm{R}$ & 0.83 & 30 & 63 & UCD \\
\hline & 2017-09-06 & 0.4-m/Boyden & 8003.49825 & $\mathrm{~V}, \mathrm{R}, \mathrm{I}$ & 0.21 & 30 & 18 & UCD \\
\hline & 2017-09-07 & 0.4-m/Boyden & 8004.29569 & $\mathrm{~B}, \mathrm{~V}, \mathrm{R}, \mathrm{I}, \mathrm{C}$ & 4.04 & 30 & 137 & UCD \\
\hline & 2017-09-08 & 0.4-m/Boyden & 8005.27849 & $\mathrm{~B}, \mathrm{~V}, \mathrm{R}, \mathrm{I}, \mathrm{C}$ & 4.42 & 30 & 491 & UCD \\
\hline & 2017-09-09 & 0.4-m/Boyden & 8006.28780 & $\mathrm{~B}, \mathrm{~V}, \mathrm{R}, \mathrm{I}, \mathrm{C}$ & 0.75 & 30 & 69 & UCD \\
\hline & 2017-09-10 & 0.4-m/Boyden & 8007.28693 & $\mathrm{~B}, \mathrm{~V}, \mathrm{R}, \mathrm{I}, \mathrm{C}$ & 2.63 & 30 & 101 & UCD \\
\hline & 2017-09-11 & 0.4-m/Boyden & 8008.28491 & $\mathrm{~B}, \mathrm{~V}, \mathrm{R}, \mathrm{I}, \mathrm{C}$ & 2.99 & 30 & 164 & UCD \\
\hline & 2017-09-12 & 0.4-m/Boyden & 8009.29553 & V, R, I, C & 2.13 & 30 & 68 & UCD \\
\hline & 2017-09-13 & 0.4-m/Boyden & 8010.29588 & $\mathrm{~B}, \mathrm{~V}, \mathrm{R}, \mathrm{I}, \mathrm{C}$ & 2.56 & 30 & 265 & UCD \\
\hline & 2017-09-14 & 0.4-m/Boyden & 8011.27748 & $\mathrm{~B}, \mathrm{~V}, \mathrm{R}, \mathrm{I}, \mathrm{C}$ & 3.01 & 30 & 328 & UCD \\
\hline & 2017-09-16 & 0.4-m/Boyden & 8013.29366 & $\mathrm{~B}, \mathrm{~V}, \mathrm{R}, \mathrm{I}, \mathrm{C}$ & 0.36 & 30 & 46 & UCD \\
\hline & 2017-09-17 & 0.4-m/Boyden & 8014.29403 & $\mathrm{~B}, \mathrm{~V}, \mathrm{R}, \mathrm{I}, \mathrm{C}$ & 1.24 & 30 & 117 & UCD \\
\hline & 2017-09-18 & 0.4-m/Boyden & 8015.29440 & $\mathrm{~B}, \mathrm{~V}, \mathrm{R}, \mathrm{I}, \mathrm{C}$ & 0.57 & 30 & 66 & UCD \\
\hline & 2017-09-19 & 0.4-m/Boyden & 8016.27751 & $\mathrm{~B}, \mathrm{~V}, \mathrm{R}, \mathrm{I}, \mathrm{C}$ & 1.13 & 30 & 129 & UCD \\
\hline & 2017-09-20 & 0.4-m/Boyden & 8017.29563 & $\mathrm{~B}, \mathrm{~V}, \mathrm{R}, \mathrm{I}, \mathrm{C}$ & 0.40 & 30 & 44 & UCD \\
\hline & 2017-09-21 & 0.4-m/Boyden & 8018.27884 & V, R, I, C & 0.20 & 30 & 19 & UCD \\
\hline & $2017-09-23$ & 0.4-m/Boyden & 8020.29042 & $\mathrm{~B}, \mathrm{~V}, \mathrm{R}, \mathrm{I}, \mathrm{C}$ & 0.76 & 30 & 87 & UCD \\
\hline & 2017-09-27 & 0.4-m/Boyden & 8024.27521 & $\mathrm{~B}, \mathrm{~V}, \mathrm{R}, \mathrm{I}, \mathrm{C}$ & 0.20 & 30 & 24 & UCD \\
\hline
\end{tabular}

* Key to observers (where UFS indicates University of the Free State): RJB (R.J. Britto, UFS), JPM (J.P. Marais, UFS), MM (M. Motsoaledi, UCT), MMN (M.M. Nyamai, UFS), AFR (A.F. Rajoelimanana, UFS),

HS (H. Szegedi, UFS), JRT (J.R. Thorstensen, Dartmouth College), UCD (Watcher, University College Dublin) 
Table 3: The spectroscopic observations. The number of exposures taken is given by $n_{\text {data }}$, the exposure time is $t_{\exp }$ and $\Delta \lambda$ gives the approximate wavelength range covered during the observation.

\begin{tabular}{|c|c|c|c|c|c|c|c|}
\hline Name & Date & $\begin{array}{l}\text { HJD (Start) } \\
(2450000+)\end{array}$ & Grating & $\begin{array}{c}n_{\text {data }} \times t_{\text {exp }} \\
(\mathrm{s})\end{array}$ & $\begin{array}{l}\Delta \lambda \\
(\AA)\end{array}$ & State & Observers* \\
\hline \multirow[t]{8}{*}{ AR Pic } & $2017-02-15$ & 7800.34472 & 7 & $2 \times 1800$ & $3900-9600$ & Quiescent & HS \\
\hline & $2017-02-18$ & 7803.27982 & 7 & $2 \times 1800$ & $3500-9200$ & N/outburst ${ }^{* *}$ & HS \\
\hline & 2017-02-19 & 7804.28084 & 7 & $1 \times 1500$ & $3500-9200$ & Fading & HS \\
\hline & 2017-02-20 & 7805.31978 & 7 & $3 \times 1500$ & $3500-9200$ & Fading & HS \\
\hline & $2017-02-21$ & 7806.27580 & 7 & $2 \times 1500$ & $3500-9200$ & Fading & HS \\
\hline & 2017-02-22 & 7807.35029 & 4 & $1 \times 600,6 \times 900$ & $3900-5200$ & Fading & JPM \\
\hline & $2017-02-23$ & 7808.43765 & 7 & $1 \times 1800$ & $3600-9300$ & Quiescent & JPM \\
\hline & $2017-02-26$ & 7811.27429 & 4 & $13 \times 1000$ & $3900-5200$ & Quiescent & JPM \\
\hline \multirow[t]{7}{*}{ QW Ser } & 2016-08-04 & 7605.24660 & 7 & $2 \times 1800$ & $2800-8500$ & Quiescent & AO, HS \\
\hline & 2016-08-08 & 7609.21541 & 4 & $11 \times 600$ & $3800-5100$ & N/outburst & AO, HS \\
\hline & 2016-08-09 & 7610.22645 & 4 & $16 \times 600$ & $3800-5100$ & Fading & AO, HS \\
\hline & 2016-08-10 & 7611.24183 & 4 & $14 \times 600$ & $3800-5100$ & Fading & AFR, HS \\
\hline & $2016-08-15$ & 7616.24744 & 7 & $2 \times 1800$ & $3450-9150$ & Quiescent & AFR, HS \\
\hline & $2017-02-23$ & 7808.59821 & 7 & $2 \times 1800$ & $3650-9350$ & Quiescent & HS \\
\hline & $2017-08-26$ & 7992.29860 & 7 & $1 \times 1800$ & $3600-9200$ & Quiescent & JPM \\
\hline \multirow[t]{8}{*}{ V521 Peg } & 2016-08-09 & 7610.47551 & 4 & $4 \times 1300$ & $3800-5100$ & Quiescent & $\mathrm{AO}, \mathrm{HS}$ \\
\hline & $2017-08-27$ & 7993.42275 & 7 & $3 \times 1200$ & $3600-9200$ & Quiescent & JPM \\
\hline & 2017-09-01 & 7998.38571 & 7 & $7 \times 400,1 \times 1200$ & $3600-9200$ & S/outburst ${ }^{* * *}$ & JPM \\
\hline & 2017-09-02 & 7999.38682 & 4 & $21 \times 600$ & $3900-5200$ & S/outburst & JPM \\
\hline & 2017-09-03 & 8000.38661 & 7 & $2 \times 400$ & $3600-9200$ & S/outburst & JPM \\
\hline & 2017-09-03 & 8000.43564 & 4 & $23 \times 400$ & $3900-5200$ & S/outburst & JPM \\
\hline & 2017-09-04 & 8001.38048 & 7 & $3 \times 400$ & $3600-9200$ & S/outburst & JPM \\
\hline & 2017-09-04 & 8001.42750 & 4 & $3 \times 400$ & $3900-5200$ & S/outburst & JPM \\
\hline
\end{tabular}

\footnotetext{
*Key to observers: JPM (J.P. Marais), AO (A. Odendaal), AFR (A.F. Rajoelimanana), HS (H. Szegedi)

${ }^{* *} \mathrm{~N} /$ outburst $=$ Normal outburst

*** S/outburst $=$ Superoutburst
}

Standard procedures, including bias, dark and flat-field correction, were applied to all the data using IRAF routines. For the spectroscopy data, cosmic ray removal, wavelength and flux calibration, and spectral extraction were performed by applying the standard IRAF routines. The spectrum given for each source per night in this paper, is an average of all the spectra taken during that night.

The standard IRAF aperture photometry routines, as well as differential photometry, were applied to the photometric data. To compile composite light curves for QW Ser and V521 Peg, a magnitude offset was determined for each filter and added to the differential magnitude values. These offset values were calculated by using the average offset of the comparison stars' magnitude values from their V and $\mathrm{i}^{\prime}$ colors as specified in the NOMAD Catalog [42] and the URAT1 Catalog [43], respectively. The magnitude offset values for the $\mathrm{V}$ filter were applied to the $\mathrm{C}$ filter differential magnitude values. The i'-filter data of QW Ser, obtained with the Watcher telescope, was plotted with the C-filter data, obtained with the UFS-Boyden telescope, as the light curves of the different filters have the same shape on the same magnitude level.

The Barycentric Julian Date (BJD) in the Barycentric Dynamical Time standard (TDB) at the 
middle of each exposure was calculated by using the 'UTC2BJD' Time Utility of the Ohio State University $^{2}$ [44]. Subsequently, the time axes of the light curve of AR Pic was converted to orbital phase. The known orbital ephemeris of AR Pic (see section 2.1), was converted to BJD $\mathrm{TDB}_{\text {before }}$ applying it to the light curve.

\section{Results}

The spectroscopic and photometric results of AR Pic, QW Ser and V521 Peg are discussed in this section. Please note that only a representative selection of the observed spectra (as listed in Table 3) is shown in this paper, since the rest of the spectra exhibited similar characteristics. The $\oplus$ symbol in the spectral plots marks the atmospheric A- and B-bands.

\subsection{AR Pic}

AR Pic was observed on 15 February 2017 during quiescence and displayed a typical DN spectrum with $\mathrm{H}, \mathrm{He}$ I and Fe II emission lines (Figure 1). The emission line profiles are double-peaked due to the Keplerian flow of the material in the accretion disc. The presence of an accretion disc is therefore confirmed. The distinguishable double peaks also indicate that the system does not have a low inclination. No features of the WD or donor star were detected. On 18 February 2017, the source was observed during a normal outburst. Although it is not clear when the source went into outburst, it was $\sim 3.3$ magnitudes brighter than in quiescence. It decreased in brightness during the following nights and by 23 February 2017 it was in quiescence again. Figure 2 illustrates how the line profile of the $\mathrm{H} \alpha$ emission line changed from double-peaked, in quiescence, to single peaked in outburst. The outburst region, and possibly an outflow, therefore dominated the emission and was much brighter than the disc. The velocity of the disc material close to the WD is approximately $2000 \mathrm{~km} \mathrm{~s}^{-1}$.

The quiescent spectra, obtained on 26 February 2017 (Figure 3), illustrate the changing asymmetry of the double-peak profile of the $\mathrm{H} \beta$ line. This demonstrates how the hot spot rotates with the orbital period. The hot spot is clearly visible at phase 0.3 (blue peak stronger) and at phase 0.8 (red peak stronger).

The strong flickering in the light curve (Figure 4), also observed by Tappert et al. [25], is evidence of a very active hot spot and the magnetic eddies that formed in the disc. There is no correlation between the orbital phase and the flickering as the flickering does not increase or decrease at any point in the orbit. No eclipse is therefore observed, which is compatible with an intermediate inclination.

\subsection{QW Ser}

QW Ser was observed spectroscopically pre-outburst (4 August 2016), during outburst (8 August 2016) and followed until it returned to quiescence (9, 10 and 15 August 2016). The quiescent spectra are that of a typical DN with double-peaked H and He I emission lines (Figure 5 (a)). During the normal outburst, the region around the WD expanded, creating a hot central region surrounded by a cooler gas. This resulted in the formation of absorption lines as emission from the

\footnotetext{
${ }^{2} \mathrm{http}$ //astroutils.astronomy.ohio-state.edu/time/utc2bjd.html
} 

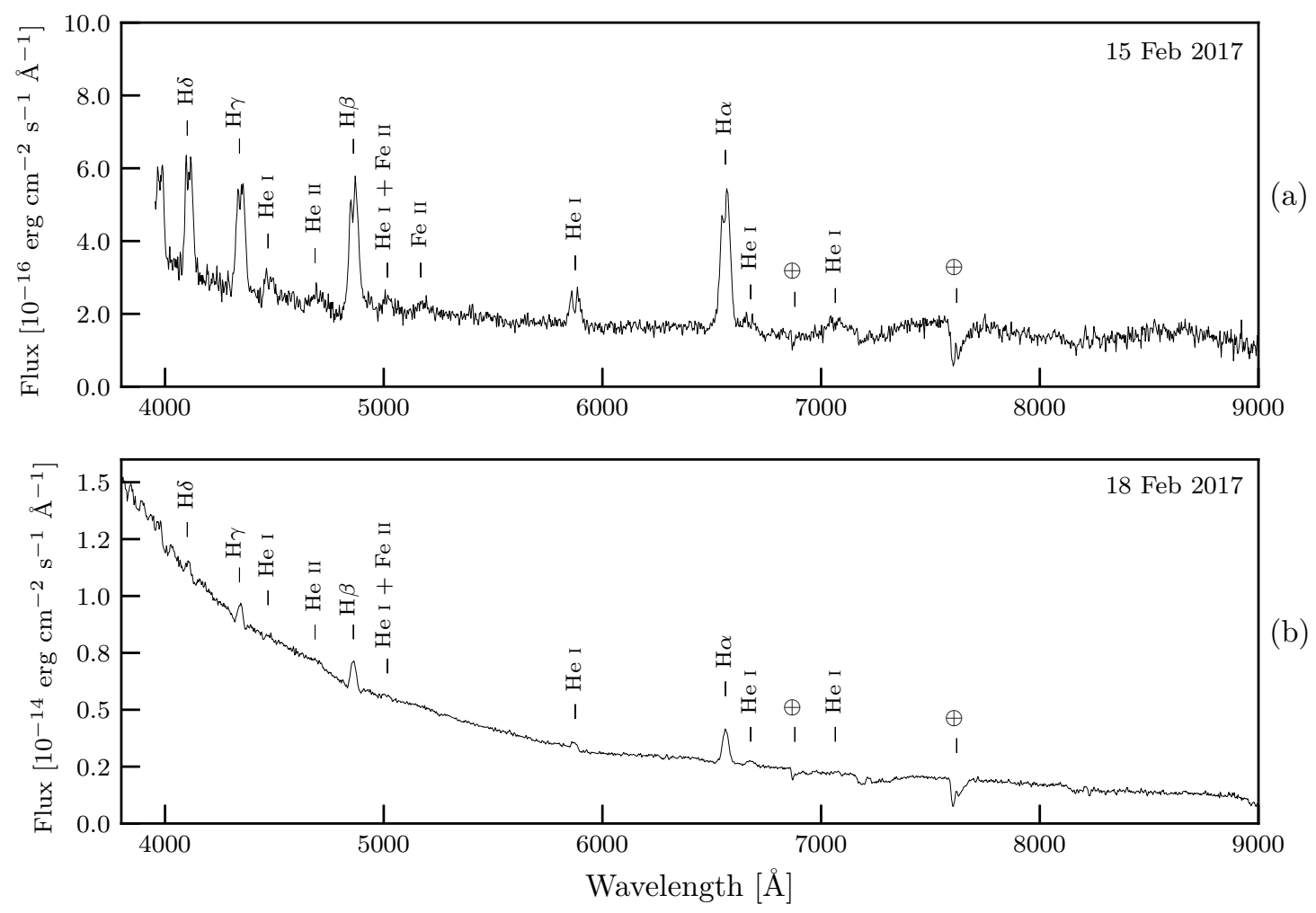

Figure 1: Spectra of AR Pic obtained (a) during quiescence on 15 February 2017, and (b) during a normal outburst on 18 February 2017.

optically thick inner (hot) region was absorbed by the surrounding gas (Figure 5 (b)). The outflows reached velocities of $\sim 1000 \mathrm{~km} \mathrm{~s}^{-1}$. As the source returned to quiescence, the disc cooled and became optically thin, allowing for the emission lines to become visible again.

The composite light curve in Figure 6 shows that QW Ser underwent a brightness increase of $\sim 5$ magnitudes and returned to quiescence within a week. During and three days after the outburst (8-11 August 2016), no significant variability was detected, apart from the post-outburst brightness decline. On 12 and 14 August 2016, the orbital modulation returned. In quiescence, two humps of unequal amplitudes were detected during one orbital modulation. This suggests an observable hot spot. Further photometric observations in quiescence are required to establish an orbital ephemeris for this source.

\subsection{V521 Peg}

The quiescent spectrum of V521 Peg in Figure 7 (a), was obtained on 27 August 2017. The spectrum displays the same characteristic absorption troughs, in which the $\mathrm{H} \beta, \mathrm{H} \gamma$ and $\mathrm{H} \delta$ lines are embedded, as observed by Rodríguez-Gil et al [35]. The He I and Fe II emission lines are also present. The double-peaked emission lines provide proof of a well-formed accretion disc and no absorption features from the secondary are detected.

Spectroscopic observations on 1 September 2017 (Figure 7 (b)) indicated that the source was in outburst, as deduced from a brightness increase of $\sim 4.5 \mathrm{mag}$, the presence of broad Balmer ab- 


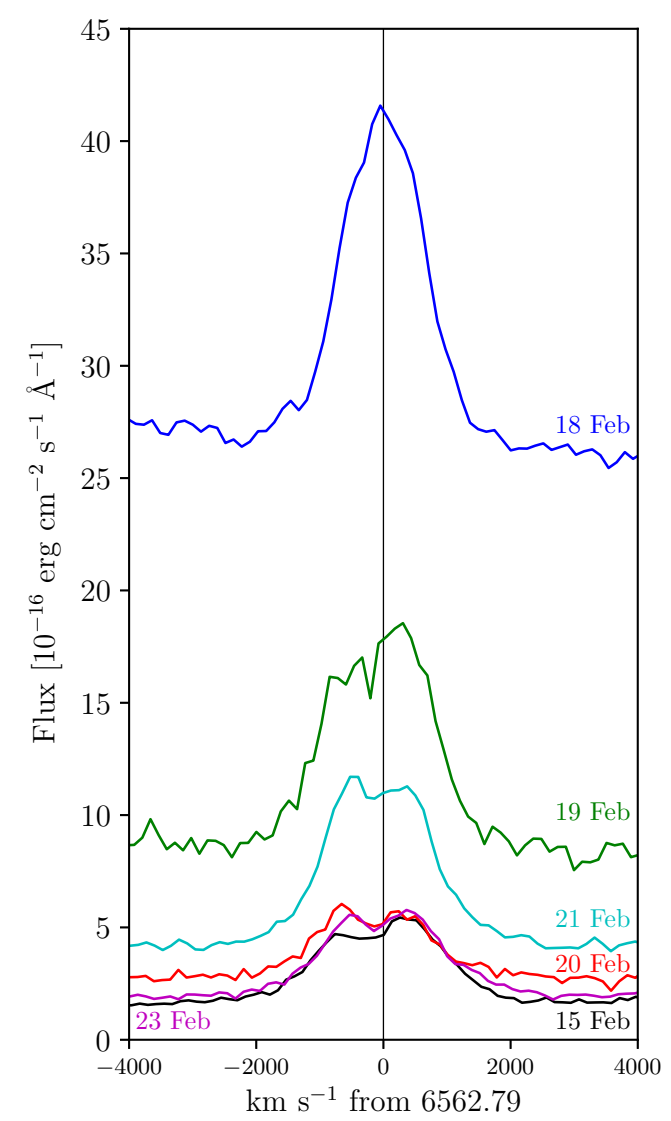

Figure 2: The $\mathrm{H} \alpha$ emission lines in the spectra of AR Pic, taken from 15 February to 23 February 2017. In quiescence the line is doublepeaked due to the Keplerian flow of the material in the accretion disc. During outburst, on 18 February 2017, the line was single peaked, indicating that the outburst region dominated the emission and was much brighter than the accretion disc. The disc material close to the WD reached velocities of $\sim 2000 \mathrm{~km} \mathrm{~s}^{-1}$.

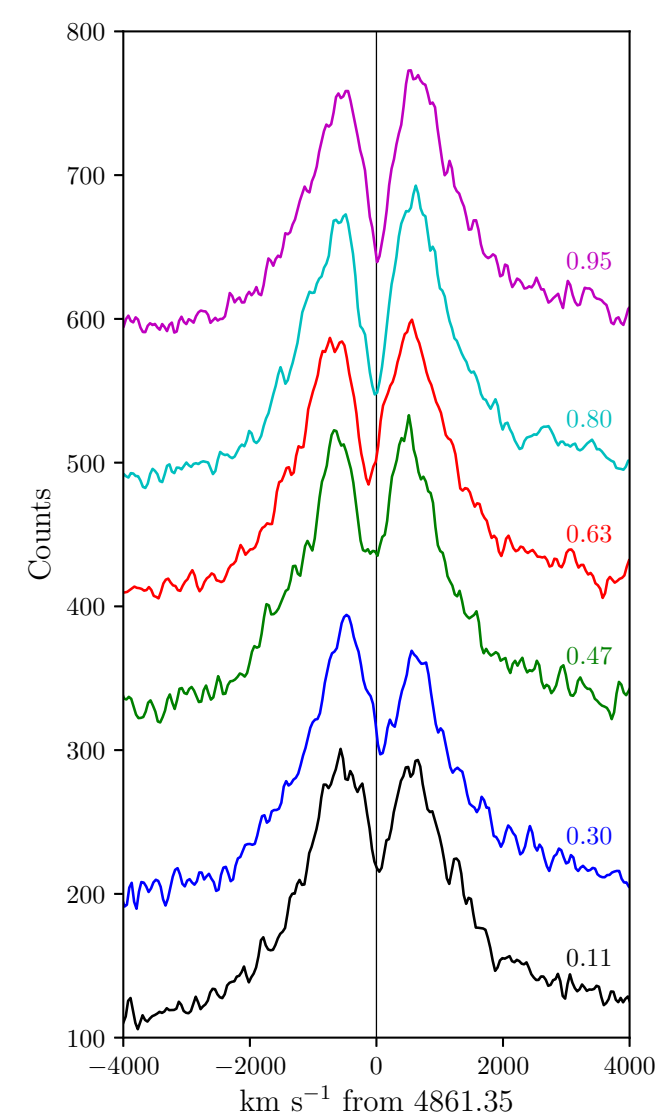

Figure 3: The $\mathrm{H} \beta$ emission lines in combined spectra of AR Pic, taken on 26 February 2017. The changing asymmetry of the doublepeak profile is evident of the hot spot rotating with the orbital period. The hot spot is clearly visible at phase 0.3 (blue peak stronger) and at phase 0.8 (red peak stronger). The lines are offset with an artificial value and plotted in phase order. The orbital phase value associated with each spectrum is indicated on the right.

sorption lines, except for $\mathrm{H} \alpha$, and a significantly bluer energy distribution. The superhumps in the light curve of the photometric observations on 2 September 2017, confirmed that it was a superoutburst. Simultaneous spectroscopic and photometric observations were conducted for the following three nights and photometric observations continued for 23 days thereafter. The composite light curve in Figure 8 shows that observations started in the plateau phase of the superoutburst. It is not possible to determine from our data when the outburst started or the exact duration, but the outburst lasted for more than 8 days.

The superoutburst spectral results (Figure 7 (b)) are comparable with spectral results of DNe WZ Sge (2001 superoutburst) [45] and GW Lib (2007 superoutburst) [46] and is typical of an optically thick accretion disc with the temperature decreasing toward its surface. $\mathrm{H} \alpha$ is almost 


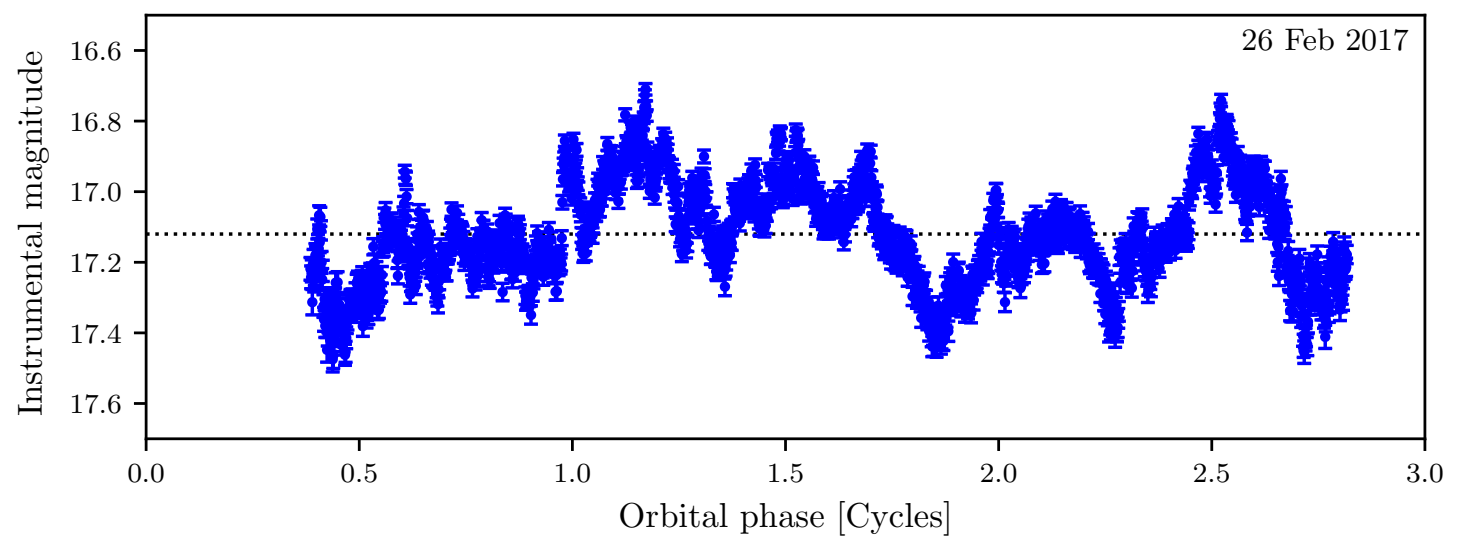

Figure 4: The light curve of AR Pic obtained on 26 February 2017. The prominent flickering in the light curve are attributed to the hot spot and magnetic eddies formed in the disc.
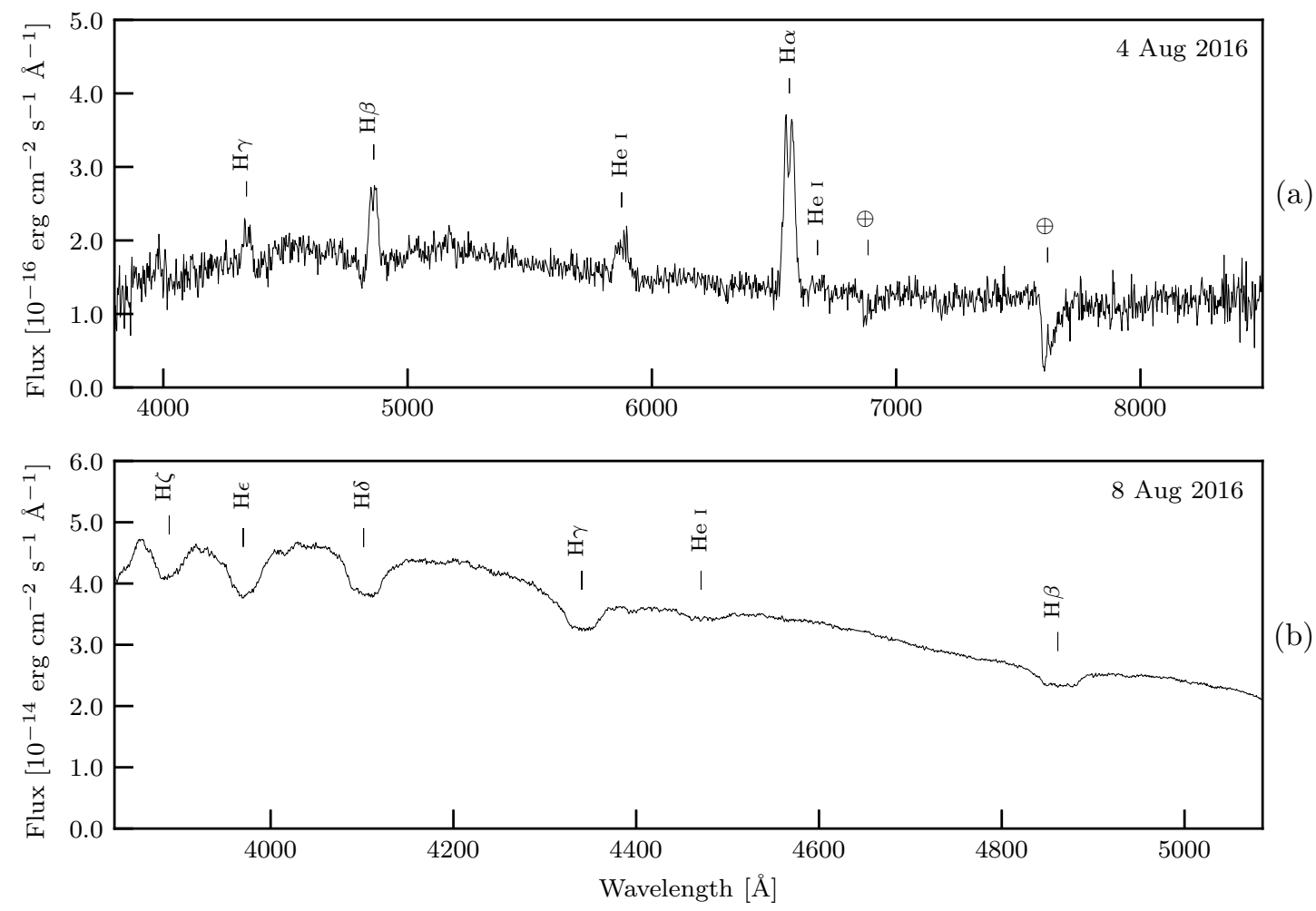

Figure 5: Spectra of QW Ser obtained (a) during quiescence on 4 August 2016, and (b) a normal outburst detected on 8 August 2016. The double-peak emission lines in (a) are evident of an accretion disc. The Balmer absorption features in (b) indicate that the disc is optically thick during an outburst. 


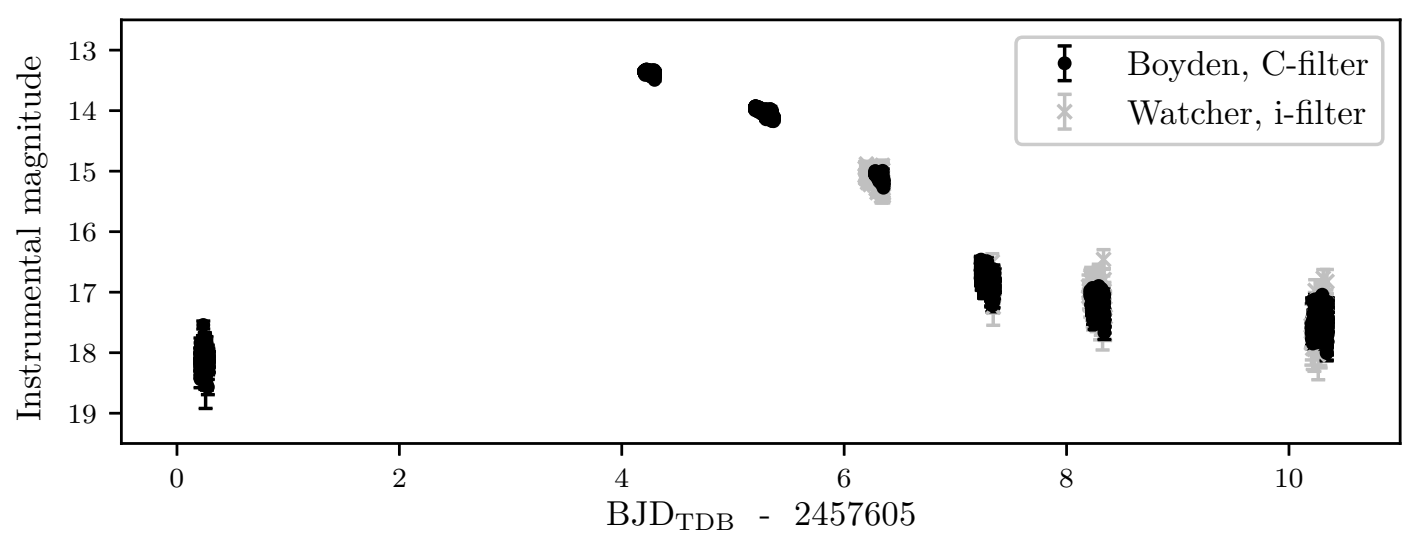

Figure 6: The composite light curve of QW Ser (4 - 14 August 2016). The system underwent a brightness increase of $\sim 5$ magnitudes during outburst and returned to quiescence within a week. Data obtained with the UFS-Boyden 1.5-m telescope (C-filter) is indicated with black dots. Data obtained with the Watcher robotic telescope (i-filter) is indicated with light gray crosses.

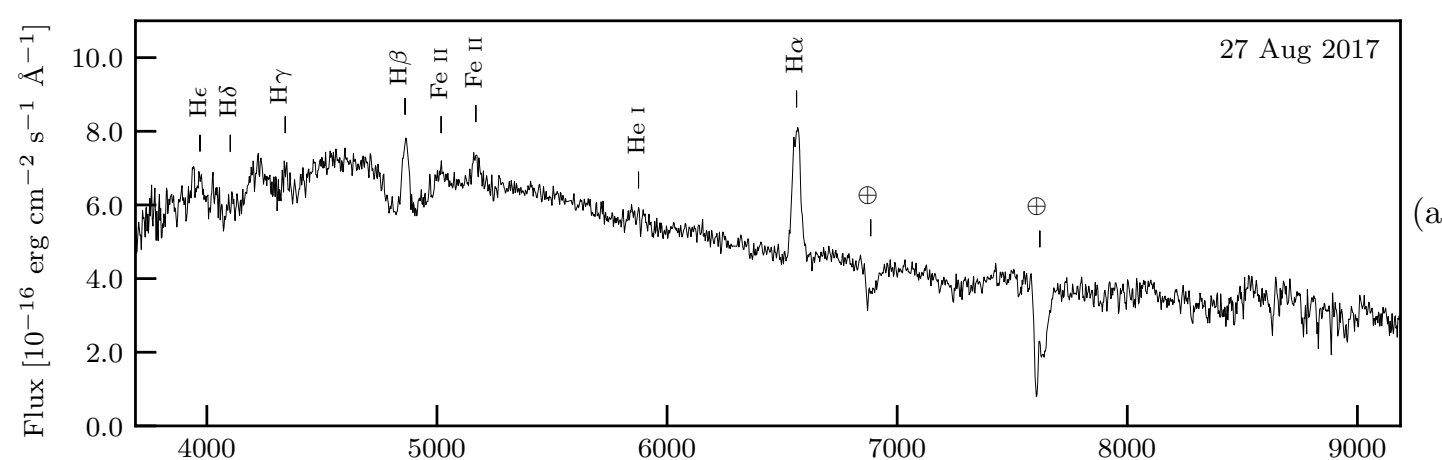

(a)

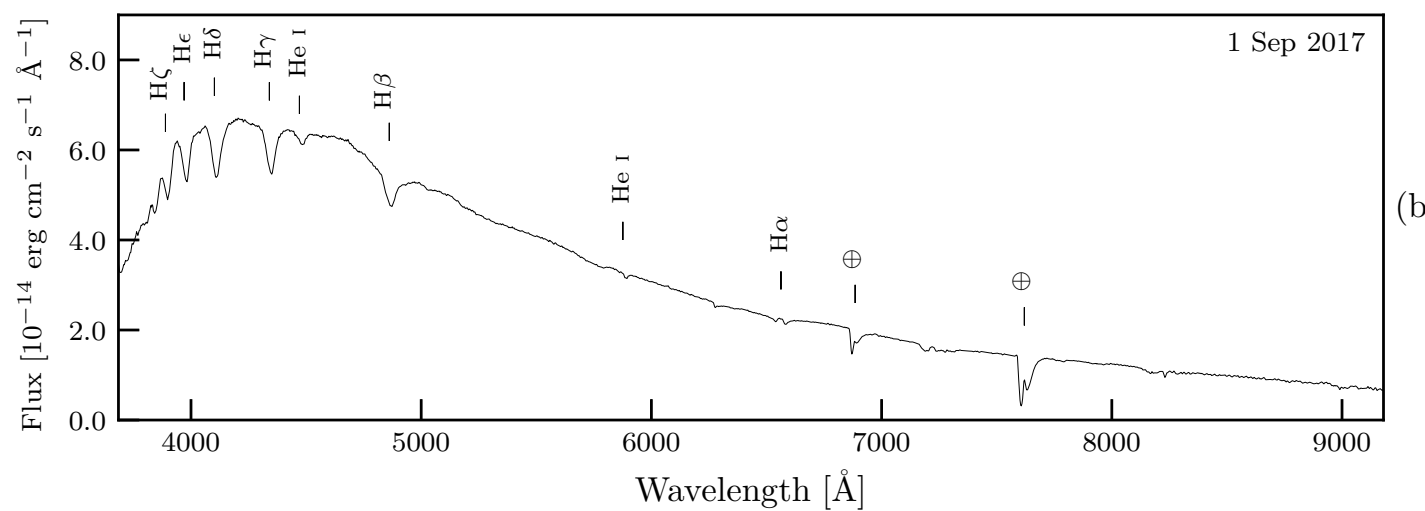

Figure 7: Spectra of V521 Peg obtained (a) during quiescence on 27 August 2017, and (b) in superoutburst on 1 September 2017. The absorption features in (b) indicate that the disc is optically thick during a superoutburst. $\mathrm{H} \alpha$ is almost undetectable as it consists of an absorption line with a superimposed emission component. 


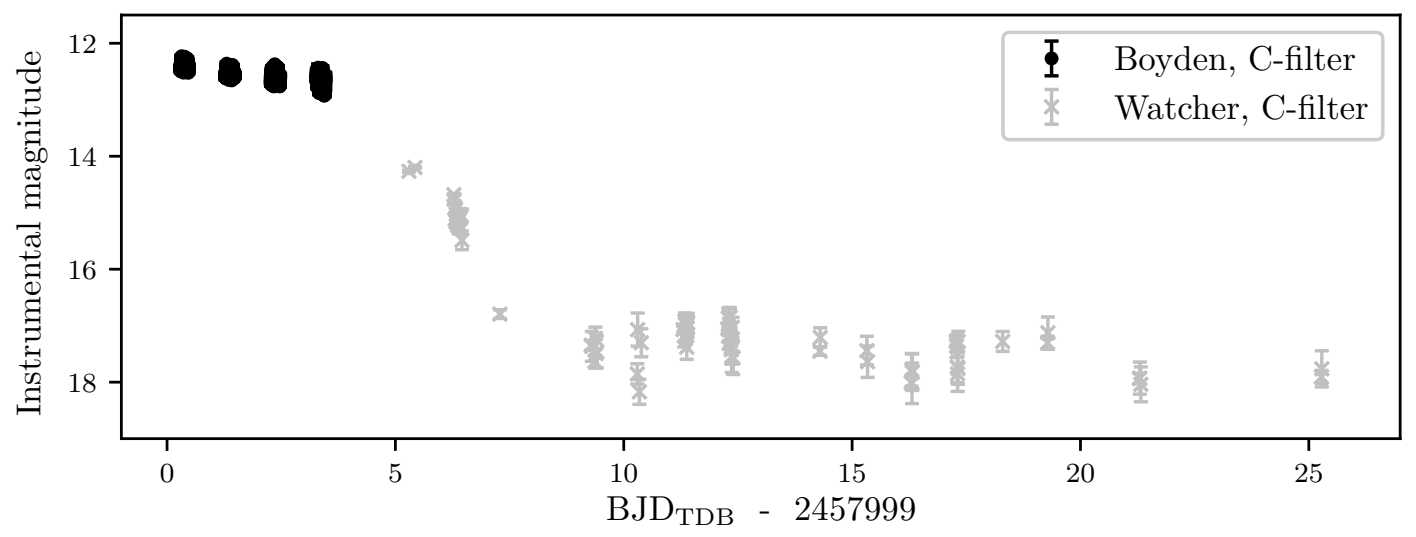

Figure 8: The composite light curve of V521 Peg (2 - 27 September 2017), indicating that the observations started in the plateau phase of the superoutburst. Data obtained with the UFS-Boyden 1.5-m telescope (Cfilter) is indicated with black dots. Data obtained with the Watcher robotic telescope (C-filter) is indicated with light gray crosses.

undetectable as it consists of an absorption line with a superimposed emission component. Hiroi et al. [46] explains that the emission component in the absorption feature is due to the photoionization of the outer disc caused by irradiation (increasing number of UV photons) by the WD and the inner, hotter part of the disc. The intensity of the emission component reduces with a decrease in the UV flux.

Superhumps were detected on 2, 3, 4, and 5 September 2017, during the plateau phase of the superoutburst. Lomb-Scargle periodograms were used to determine a superhump period for each observation. An average superhump period for the four observations was determined as $P_{\mathrm{sh}} \sim 1.48$ hr. This value, together with the known orbital period of $P_{\text {orb }} \sim 1.44 \mathrm{hr}$, relates to a period excess of $\varepsilon \sim 3 \%$ (From Eq. 1.4). The calculated period excess was used to determine a mass ratio of $q \sim 0.14$ (From Eq. 1.5) and confirms the mass ratio determined by Rodríguez-Gil et al [35].

\section{Conclusion}

We reported on the quiescent states and normal outbursts detected for AR Pic and QW Ser, and a superoutburst of V521 Peg. All the sources showcased typical SU UMa-type dwarf nova characteristics in the optical photometry and spectroscopy.

AR Pic had a magnitude increase of $\sim 3.3$ but returned to quiescence fairly quickly. Strong flickering was detected, which is attributed to a very active hot spot and magnetic eddies formed in the accretion disc. The hot spot was also observable in the asymmetric double-peaked $\mathrm{H} \beta$ emission line. The disc material, close to the WD, reached velocities of $\sim 2000 \mathrm{~km} \mathrm{~s}^{-1}$.

During outburst, QW Ser had a brightness increase of $\sim 5$ magnitudes. The flat base of the absorption lines in the spectra indicated that the outflows reached velocities of $\sim 1000 \mathrm{~km} \mathrm{~s}^{-1}$. The system returned to quiescence within a week. Observations during quiescence suggest an observable hot spot. 
Optical spectra of V521 Peg during superoutburst were obtained and presented for the first time. The spectra exhibited the same characteristics of other SU UMa DNe, WZ Sge and QW $\mathrm{Lib}$, during superoutburst. The most interesting feature is the $\mathrm{H} \alpha$ absorption/emission line which seems almost undetected. The absorption line, caused by the optically thick disc, has an emission component within. The emission component is from the photoionized outer disc due to irradiation from the WD and inner (hot) disc. An average superhump period of $P_{\mathrm{sh}} \sim 1.48 \mathrm{hr}$ was determined for the observed superhumps. The period excess $\varepsilon \sim 3 \%$ suggests a mass ratio of $q \sim 0.14$. The superoutburst lasted for at least 8 days and a magnitude increase of $\sim 4.5$ magnitude was detected.

\section{Acknowledgements}

This paper uses observations made at the Boyden Observatory and the South African Astronomical Observatory (SAAO). The first author would like to thank all the observers who performed simultaneous observations of the sources discussed in this paper. She expresses her appreciation towards the National Research Foundation (NRF) for the financial assistance towards this research. The CRTS survey is supported by the U.S. National Science Foundation under grants AST-0909182. The CSS survey is funded by the National Aeronautics and Space Administration under Grant No. NNG05GF22G issued through the Science Mission Directorate Near-Earth Objects Observations Program. This research has made use the SIMBAD database as well as the VizieR catalogue access tool, CDS, Strasbourg, France.

\section{References}

[1] J. Smak, Outbursts of dwarf novae, PASP 96 (1984) 5-18.

[2] B. Warner, Cataclysmic Variable Stars, vol. 28. Cambridge University Press, Cambridge, 1995.

[3] F. Giovannelli and L. Sabau-Graziati, The Golden Age of Cataclysmic Variables and Related Objects: The State of Art., in The Golden Age of Cataclysmic Variables and Related Objects - III (Golden2015), p. 1, 2015.

[4] H. Ritter and U. Kolb, Catalogue of cataclysmic binaries, low-mass X-ray binaries and related objects (update RKcat7.24, 2016), A\&A 404 (2003) 301-303.

[5] M. A. Wood, M. D. Still, S. B. Howell, J. K. Cannizzo and A. P. Smale, V344 Lyrae: A Touchstone SU UMa Cataclysmic Variable in the Kepler Field, ApJ 741 (2011) 105, [1108.3083].

[6] C. Hellier, Cataclysmic Variable Stars - How and why they vary. Praxis Publishing Ltd, Chichester, 2001.

[7] D. L. Coppejans, E. G. Körding, C. Knigge, M. L. Pretorius, P. A. Woudt, P. J. Groot et al., Statistical properties of dwarf novae-type cataclysmic variables: the outburst catalogue, MNRAS 456 (2016) 4441-4454.

[8] R. Whitehurst, Numerical simulations of accretion disks. I - Superhumps - A tidal phenomenon of accretion disks, MNRAS 232 (1988) 35-51.

[9] N. Vogt, The SU UMa stars - an important sub-group of dwarf novae, A\&A 88 (1980) 66-76.

[10] J. Patterson, Eruptions and superhumps in dwarf novae, AJ 84 (1979) 804-811. 
[11] M. Hirose and Y. Osaki, Hydrodynamic simulations of accretion disks in cataclysmic variables Superhump phenomenon in SU UMa stars, PASJ 42 (1990) 135-163.

[12] S. A. Balbus and J. F. Hawley, Instability, Turbulence, and Enhanced Transport in Accretion Disks, in IAU Colloq. 163: Accretion Phenomena and Related Outflows (D. T. Wickramasinghe, G. V. Bicknell and L. Ferrario, eds.), vol. 121 of Astronomical Society of the Pacific Conference Series, p. 90, 1997.

[13] E. Priest and T. Forbes, eds., Magnetic reconnection : MHD theory and applications, 2000.

[14] A. Odendaal and P. Meintjes, Cataclysmic variables: New frontiers in multi-wavelength research, in Proceedings of the 4th Annual Conference on High Energy Astrophysics in Southern Africa (HEASA 2016). January 13th, 2016. South African Astronomical Observatory (SAAO), Cape Town, South Africa., p. 30, 2016.

[15] P. J. Meintjes and E. Breedt, Magnetic viscosity: outbursts and outflows in accretion driven systems , Mem.S.A.It. 86 (2015) 89.

[16] S. Scaringi, T. J. Maccarone, C. D’Angelo, C. Knigge and P. J. Groot, Magnetically gated accretion in an accreting 'non-magnetic' white dwarf, Nature 552 (2017) 210-213, [1712 . 04949 ].

[17] P. Szkody, The minimum state of dwarf novae, ApJ 207 (1976) 824-836.

[18] Y. Osaki, A model for the superoutburst phenomenon of SU Ursae Majoris stars, PASJ 41 (1989) 1005-1033.

[19] Y. Osaki, Irradiation-induced mass-overflow instability as a possible cause of superoutbursts in $S U$ UMa stars, A\&A 144 (1985) 369-380.

[20] E. Breedt, B. T. Gänsicke, A. J. Drake, P. Rodríguez-Gil, S. G. Parsons, T. R. Marsh et al., 1000 cataclysmic variables from the Catalina Real-time Transient Survey, MNRAS 443 (2014) 3174-3207.

[21] A. J. Drake, S. G. Djorgovski, A. Mahabal, E. Beshore, S. Larson, M. J. Graham et al., First Results from the Catalina Real-Time Transient Survey, ApJ 696 (2009) 870-884.

[22] G. Pojmanski, The All Sky Automated Survey. Catalog of Variable Stars. I. 0 h - 6 hQuarter of the Southern Hemisphere, Acta Astronomica 52 (2002) 397-427, [ast ro-ph/ 0210283 ].

[23] F. Genova, D. Egret, O. Bienaymé, F. Bonnarel, P. Dubois, P. Fernique et al., The CDS information hub. On-line services and links at the Centre de Données astronomiques de Strasbourg, A\&AS 143 (2000) 1-7.

[24] J. Maza, M. T. Ruiz, L. E. Gonzalez and M. Wischnjewsky, Calan-Tololo survey. I - Seyfert 1 galaxies, ApJS 69 (1989) 349-352.

[25] C. Tappert, T. Augusteijn and J. Maza, Cataclysmic variables from the Calán-Tololo Survey - I. Photometric periods, MNRAS 354 (2004) 321-331, [astro-ph / 0408357 ].

[26] A. Imada, T. Kato, L. A. G. B. Monard, R. Stubbings, M. Uemura, R. Ishioka et al., Photometric Studies of New Southern SUUMa-Type Dwarf Novae, FL Trianguli Australis and CTCV J0549-4921, PASJ 60 (2008) 267-273, [0804.3126].

[27] T. Kato, A. Imada, M. Uemura, D. Nogami, H. Maehara, R. Ishioka et al., Survey of Period Variations of Superhumps in SU UMa-Type Dwarf Novae, PASJ 61 (2009) S395-S616, [0 905.1757 ].

[28] K. Takamizawa, , VSOLJ Variable Star Bulletin 30 (1998) 3.

[29] P. Schmeer, , vsnet-alert 3548 (1999) 3. 
[30] D. Nogami, M. Uemura, R. Ishioka, H. Iwamatsu, T. Kato, E. P. Pavlenko et al., A New SU UMa-Type Dwarf Nova, QW Serpentis (= TmzV46), PASJ 56 (2004) S99-S107, [astro-ph/ 0310274 ].

[31] A. Olech, P. Kędzierski, K. Złoczewski, K. Mularczyk and M. Wiśniewski, Curious Variables Experiment (CURVE) CCD photometry of $Q W$ Serpentis in superoutburst and quiescence, $A \& A 411$ (2003) 483-487, [astro-ph/ 0307497 ].

[32] J. Patterson, J. Kemp, D. A. Harvey, R. E. Fried, R. Rea, B. Monard et al., Superhumps in Cataclysmic Binaries. XXV. $q_{\text {crit }}, \varepsilon(q)$, and Mass-Radius, PASP 117 (2005) 1204-1222, [astro-ph/ 0507371 ].

[33] K. J. Pearson, Superhumps: confronting theory with observation, MNRAS 371 (2006) 235-244, [astro-ph/0605743].

[34] B. T. Gänsicke, H.-J. Hagen and D. Engels, Properties of a spectroscopically selected CV sample, in The Physics of Cataclysmic Variables and Related Objects (B. T. Gänsicke, K. Beuermann and K. Reinsch, eds.), vol. 261 of Astronomical Society of the Pacific Conference Series, p. 190, 2002, astro-ph/0111375.

[35] P. Rodríguez-Gil, B. T. Gänsicke, H.-J. Hagen, T. R. Marsh, E. T. Harlaftis, S. Kitsionas et al., Detection of the white dwarf and the secondary star in the new SU UMa dwarf nova $<A S T R O B J>H S$ 2219+1824</ASTROBJ>, A\&A 431 (2005) 269-277, [astro-ph/ 0409668 ].

[36] T. Kato, F.-J. Hambsch, H. Maehara, G. Masi, F. Nocentini, P. A. Dubovsky et al., Survey of period variations of superhumps in SU UMa-type dwarf novae. V. The fifth year (2012-2013), PASJ 66 (2014) 30, [1310.7069].

[37] T. Kato, P. A. Dubovsky, I. Kudzej, F.-J. Hambsch, I. Miller, T. Ohshima et al., Survey of period variations of superhumps in SU UMa-type dwarf novae. VI. The sixth year (2013-2014), PASJ 66 (2014) 90, [1406.6428].

[38] A. Ferrero, L. Hanlon, R. Felletti, J. French, G. Melady, S. McBreen et al., The Photometry Pipeline of the Watcher Robotic Telescope, Advances in Astronomy 2010 (2010) 715237.

[39] D. Murphy, "Watcher: A hardware guide." http://watchertelescope.ie/files/Watcher_Hardware.pdf, 2014.

[40] R. Coppejans, A. A. S. Gulbis, M. M. Kotze, D. L. Coppejans, H. L. Worters, P. A. Woudt et al., Characterizing and Commissioning the Sutherland High-Speed Optical Cameras (SHOC), PASP 125 (2013) 976.

[41] L. A. Crause, D. Carter, A. Daniels, G. Evans, P. Fourie, D. Gilbank et al., SpUpNIC (Spectrograph Upgrade: Newly Improved Cassegrain) on the South African Astronomical Observatory's 74-inch telescope, in Ground-based and Airborne Instrumentation for Astronomy VI, vol. 9908 of Proceedings of the SPIE, p. 990827, 2016, DOI.

[42] N. Zacharias, D. G. Monet, S. E. Levine, S. E. Urban, R. Gaume and G. L. Wycoff, VizieR Online Data Catalog: NOMAD Catalog (Zacharias+ 2005), VizieR Online Data Catalog 1297 (2005).

[43] N. Zacharias, C. Finch, J. Subasavage, G. Bredthauer, C. Crockett, M. Divittorio et al., The First U.S. Naval Observatory Robotic Astrometric Telescope Catalog, AJ 150 (2015) 101, [1508. 04637 ].

[44] J. Eastman, R. Siverd and B. S. Gaudi, Achieving Better Than 1 Minute Accuracy in the Heliocentric and Barycentric Julian Dates, PASP 122 (2010) 935, [1005.4415].

[45] D. Nogami and T. Iijima, Dramatic Spectral Evolution of WZ Sagittae during the 2001 Superoutburst, PASJ 56 (2004) S163-S182, [astro-ph/ 0401292 ]. 
[46] K. Hiroi, Y. Moritani, D. Nogami, A. Imada, O. Hashimoto, Y. Ueda et al., Spectroscopic Observations of the WZ Sge-Type Dwarf Nova GW Librae during its 2007 Superoutburst, PASJ 61 (2009) 697-705, [0904.3425]. 